Research Article

\title{
Two-Stroke Thermodynamic Cycle Optimization of a Single-Cylinder Free-Piston Engine Generator
}

\author{
Houliang Yu, Zhaoping Xu (D), Qinglin Zhang, Liang Liu, and Ru Hua \\ School of Mechanical Engineering, Nanjing University of Science and Technology, Nanjing 210094, China \\ Correspondence should be addressed to Zhaoping Xu; xuzhaoping@njust.edu.cn
}

Received 4 March 2019; Accepted 7 April 2019; Published 2 May 2019

Academic Editor: Fuat Kara

Copyright (C) 2019 Houliang Yu et al. This is an open access article distributed under the Creative Commons Attribution License, which permits unrestricted use, distribution, and reproduction in any medium, provided the original work is properly cited.

\begin{abstract}
A free-piston engine generator (FPEG) is a new type of energy converter, which eliminates the crankshaft and connecting rod mechanism. In order to achieve efficient energy conversion, the two-stroke thermodynamic performance optimization of a singlecylinder free-piston engine generator is investigated in this paper. Firstly, the components, four-stroke thermodynamic cycle, twostroke thermodynamic cycle, and prototype system of the FPEG are presented in detail. The one-dimensional flow simulation model of the FPEG is created based on the gas dynamics equation, Weber combustion function, and heat transfer function, and then the model is validated by the data tested from the prototype system. According to the four-stroke experimental results of the FPEG, an effective power of $4.75 \mathrm{~kW}$ and a peak pressure of 21.02 bar have been obtained. Then, the two-stroke thermodynamic cycle is simulated and compared under the different control parameters of intake air pressure, injection timing, ignition timing, and valve timing through the simulation model. The optimized results show that an indicated thermal efficiency of $27.6 \%$, an indicated power of $6.7 \mathrm{~kW}$, and a maximal working frequency of $25 \mathrm{~Hz}$ can be achieved by the prototype system, when the twostroke thermodynamic cycle is used.
\end{abstract}

\section{Introduction}

Concerns about energy saving and emissions reduction have resulted in modifications in the structure of an internal combustion engine (ICE), one method to address this issue is via the free-piston engine [1-3]. A free-piston engine generator (FPEG) is a new type of power plant, which has attracted the research interests of worldwide scholars because of its particular advantages in terms of high efficiency and low emission.

Compared with a conventional generator system, this new energy conversion device shows the superiority such as structural simplicities, low manufacturing cost, and high power. The biggest difference in structure is the elimination of the crankshaft and flywheel of engine and the piston and the mover of the linear generator are directly connected. Thus, the free-piston can oscillate between its two endpoints and be influenced by all forces acting on it. Without the limitation of the connection rod mechanism, piston movement friction dropped significantly and the FPEG structure was more compact $[4,5]$. The free-piston engine generator has the ability to accommodate multifuel by easily controlling the compression ratio, and the indicated power and system efficiency could be improved by optimizing the thermodynamic cycle.

Studies showed that most of the two-stroke free-piston engines have similar working principle. Based on the theoretical analysis, the two-stroke engine has achieved high power density and thermal efficiency. In the past decades, Clark and other researchers from West Virginia University had done much research work on the free-piston engine generator. They developed the first prototype system of a free-piston engine generator in 1998, which is a spark-ignite dual-piston structure with a cylinder bore of $36.5 \mathrm{~mm}$ and a maximum piston stroke of $50 \mathrm{~mm}[6,7]$. As reported that the prototype was working at the frequency of $23.1 \mathrm{~Hz}$, the maximum output electric power is $316 \mathrm{~W}$ and the energy conversion efficiency is $11 \%$. However, the output power and energy conversion efficiency are significantly lower than the simulated results of $50 \%$ 
Suat Sarıdemir and Fuat Kara at Düzce University developed an artificial neural network (ANN) model in order to predict the torque and power of a beta-type Stirling engine. After comparing the predicted valves of the model with the experimental results, the validity of the established ANN model is verified. They also used a multiple regression method to evaluate the prediction capability of the model, and the results showed that the ANN is a dependable model to predict the torque and power of the beta-type Stirling engine $[8,9]$.

Researchers at Toyota Central R\&D Labs Inc also developed a single-piston free-piston engine linear generator (FPEG), which consisted of an integrated combustion chamber, a gas spring chamber, and a linear generator. FPEG adopted the two-stroke working mode, and it could operate continuously for many hours. After conducting a power generation experiment on FPEG prototype system, the results demonstrated that it can realize reliable and stable operations in all the modes of start-up, motoring and firing [10].

In $[11,12]$, Xu et al. at Nanjing University of Science and Technology developed a novel single-cylinder four-stroke FPEG prototype in 2010. As an internal combustion linear generator, the prototype system achieved continuous and stable operation of the four-stroke working cycle. It is equipped with electromagnetic valve train to complete the scavenging process, Moreover, the $58 \mathrm{Nm}$ peak torque with the maximum output power of $10 \mathrm{~kW}$ has been obtained. On this basis, $\mathrm{Xu}$ proposed an improved method which optimized the two-stroke thermodynamic cycle of FPEG to achieve the thermodynamic performance of high efficiency and energy saving.

In this paper, in order to achieve the characteristic of higher power and optimize the two-stroke thermodynamic performance, an experiment system of the FPEG is established and made appropriate modifications. In the following sections, the components and working principle of middle spring-rebounded FPEG are introduced. In Section 3, the one-dimensional flow simulation model of the FPEG is built, which is validated by means of a four-stroke experiment. Then, the two-stroke thermodynamic cycle of FPEG is simulated under different influential factors, and the simulation results are compared and analyzed in detail. The optimized results will help us to understand how the twostroke thermodynamic cycle of FPEG affects the indicated power and system efficiency.

\section{Structure and Working Principle of the FPEG}

2.1. Basic Structure of the FPEG. The elementary structure of the free-piston engine generator is shown in Figure 1. The main parts of the FPEG are a gasoline engine, back spring, and linear electric generator. The system has only one combustion chamber, a rebounding device, and a reciprocating moving component. The combustion chamber is a single-cylinder free-piston engine, which is equipped with electromagnetic valves, injector, and spark plug. A back spring is assembled between the combustion chamber and linear electric generator. The single piston and the moving coil of the linear generator are connected into one compact

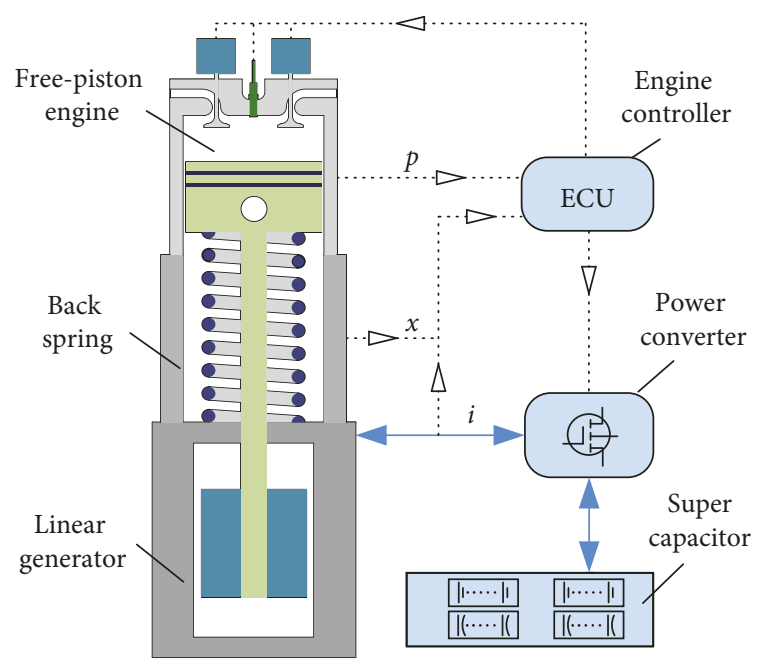

FIgURE 1: Basic configurations of FPEG.

component, as a whole mover of FPEG. The free-piston will move freely between the top dead center (TDC) and the bottom dead center (BDC), and its reciprocating motion is determined by the imbalance of all forces acting on the mover [11, 13].

The free-piston engine will operate with trapped fuel mixture and spark plug ignition. Because the generating efficiency of a linear electrical generator reduced significantly at low-speed conditions, the back spring pushes the piston upward to achieve continuous operation. A supercapacitor is used to incorporate the electricity output by the generator. The power converter is used for matching the linear generator and storing the electric energy $[14,15]$. The electronic controller unit (ECU) could control the system to adjust engine performance after acquiring the signals of cylinder pressure, piston displacement, armature current, and others. Besides, scavenging is implemented by the electromagnetic valves, which are fixed on the cylinder head. In a complete working cycle, the linear generator works in the motoring mode only in the intake stroke, while other strokes work in the generating mode.

In the FPEG system, there is a great deal of freedom in defining the piston motion. The FPEG working cycle can be switched by changing the movement law of the piston. Thus, the four-stroke thermodynamic cycle and two-stroke thermodynamic cycle can be used for different working cycles of the FPEG.

2.2. Thermodynamic Cycle of the FPEG. Four-stroke freepiston engines have relatively more energy saving and higher efficiency than two-stroke free-piston engines, but twostroke has the advantages of power density. At the same working frequency, the two-stroke working cycle number is twice that of the four-stroke, and the time of gas exchange is shorter than the four-stroke [16]. The four-stroke and twostroke thermodynamic cycles of FPEG are presented to optimize the thermodynamic performance.

As can be seen from Figure 2, the remarkable characteristics of the four-stroke thermodynamic cycle are the 


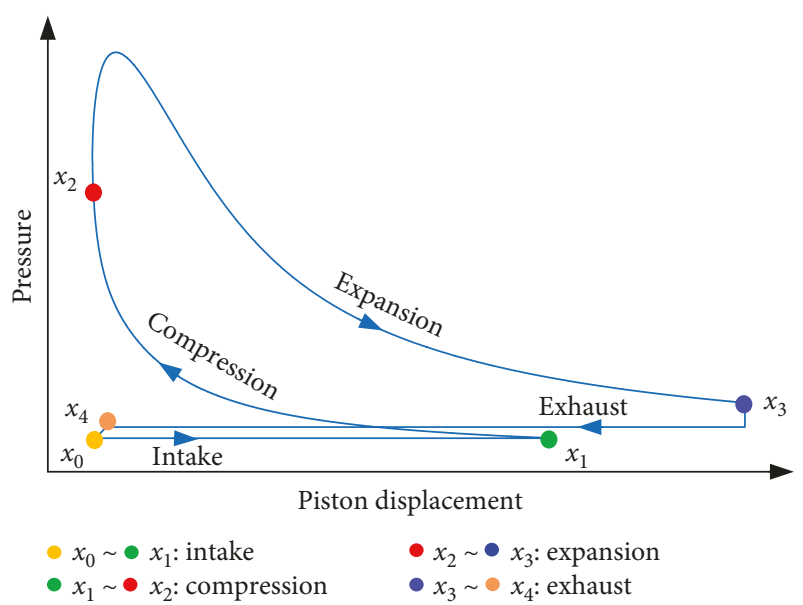

FIgUre 2: The four-stroke thermodynamic cycle of FPEG.

short intake and compression stroke, which are supplemented by pressurized the intake air [17]. During the intake stroke, the linear generator works as an electric machine to drive the piston assembly move downward from point $x_{0}$ to point $x_{1}$ to absorb the fuel mixture. It can adjust the intake pressure or air temperature to increase the mixture flow and improve the combustion process. When the piston moves to TDC and approaches the point $x_{2}$, the fuel mixture is compressed in the compression stroke. During the expansion stroke, the ignition of the spark plug is the start point for the combustion process and it will end at point $x_{3}$. After that, the piston moves from bottom to top and reaches the point $x_{4}$ to expel the burned gas. Thus, the expansion and exhaust strokes are longer than intake and compression strokes, and it can achieve the full combustion to increase the power density.

As shown in Figure 3, the two-stroke thermodynamic cycle is characterized by short compression and expansion stroke, which is supplemented by adjusting the spark advance angle to realize more full combustion. The longer valve overlap can increase the valve opening duration of the intake and exhaust strokes. Before the piston reaches the point $x_{0}$, the spark plug ignites the fuel mixture and the piston moves upward to accomplish the compression stroke. During the exhaust stroke, the piston moves from point $x_{1}$ to point $x_{3}$. Then, the piston moves from point $x_{2}$ to point $x_{4}$ in the intake stroke. When the piston moves from point $x_{2}$ to point $x_{3}$, the valve overlap realizes the intake and exhaust valves open simultaneously to absorb the fuel mixture and expel the residual gas. It can increase the volumetric efficiency and improve the process of gas exchange. Besides, the advance ignition can achieve the sufficient combustion to release more energy.

2.3. Prototype and Experiment System. The prototype structure of FPEG is described in Figure 4. The prototype is a single-piston, four-stroke, gasoline engine, which is equipped with four electromagnetic valves. It employs the watercooled cooling method, closed-loop control of intake port fuel injection, and electronically controlled spark-ignition

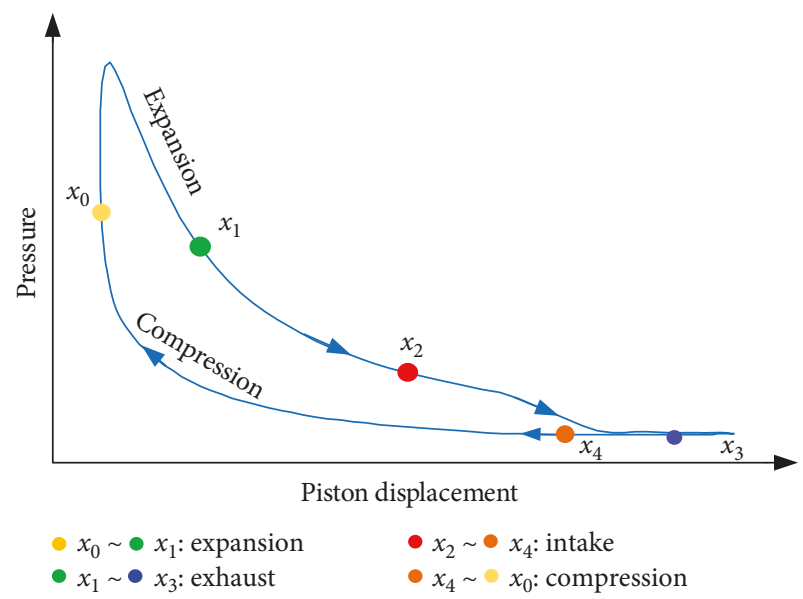

Figure 3: The two-stroke thermodynamic cycle of FPEG.

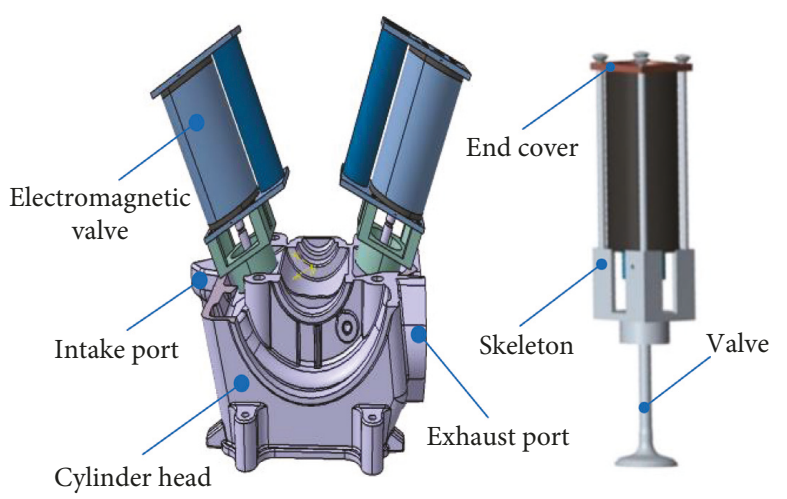

FIgURE 4: The prototype structure of FPEG.

system. Compared with the design requirements of the FPEG, the prototype performances are very consistent and facilitate to refit. Table 1 lists the main structure parameters of prototype.

The overall structure of the electromagnetic valve is shown in Figure 4. The tubular structure consists of iron core, coil skeleton, coil, permanent magnet layer, and outer wall of actuator. In the electromagnetic valve system, the coil and valve are connected rigidly, and the back spring is assembled between the coil skeleton and the cylinder head. The electromagnetic valve is used for providing the scavenging air and realizing effective control of the gas exchange process. Under the control of electronic controller unit (ECU), it can change valve lift, valve opening time, and valve opening duration, so it can achieve flexible control of the valve mechanism.

Figure 5 shows the 3-D structures of a tubular moving coil linear generator (MCLG). The MCLG is a single-phase moving-coil permanent magnet generator, also called the voice coil motor (VCM). The linear generator consists of a permanent magnet (PM), core, moving coil, and end cover. An air-gap reserved between outer core and inner core. In order to acquire high air-gap flux density, the PM adopts radial magnetization and the magnetization direction of PM-A and PM-B is opposite. The nonmagnetic coil skeleton is wound two coils, which is the whole mover of MCLG. Furthermore, the coil current is not commutated current 
TABLE 1: Specification of prototype.

\begin{tabular}{lcc}
\hline Parameters & Unit & Value \\
\hline Bore & $\mathrm{mm}$ & 102 \\
Stroke & $\mathrm{mm}$ & 126 \\
Volume & $\mathrm{cm}^{3}$ & 695 \\
Valve seat diameter & $\mathrm{mm}$ & 36 \\
Minimal top dead center & $\mathrm{mm}$ & 18 \\
Maximal bottom dead center & $\mathrm{mm}$ & 120 \\
Motor maximal displacement & $\mathrm{cc} / \mathrm{r}$ & 182 \\
Compression ratio & - & 9.3 \\
Generation efficiency of MCLG & $\%$ & 95.2 \\
Maximum thrust force of generator & $\mathrm{N}$ & 3200 \\
\hline
\end{tabular}

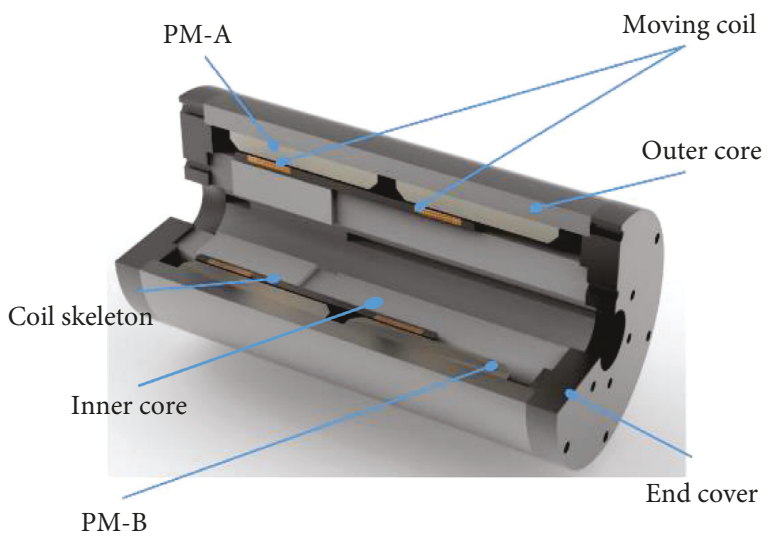

FIgURE 5: Three-dimensional structure of MCLG.

that can increase the efficiency of MCLG system. The structure has the advantages of less moving mass, fast response, and low coil inductance $[18,19]$.

Based on the components of the prototype, electromagnetic valve, moving coil linear generator, and sensors, the experiment system of FPEG has been built. As shown in Figure 6, the experiment system is used to test and validate the thermodynamic performance of the FPEG. The system also includes an engine controller and power converter, which is equipped with the cylinder pressure sensor, displacement sensor, and current sensor. The sensors can collect the information of the system at working state and transfer the information to the controller, which calculates the test results.

\section{Modeling of the FPEG}

The thermodynamic cycle of FPEG is affected by various factors such as gas dynamic, heat release process, and heat transfer loss. In this section, the simulation model of the FPEG is established based on an one-dimensional gas dynamics equation, Weber combustion function, and heat transfer function.

3.1. One-Dimensional Gas Dynamics. In order to describe the one-dimensional gas dynamics in the pipe of free-piston engine, the following points are assumed: (1) the state of the working medium in the combustion chamber is ideal homogeneous gas. (2) The temperature, pressure, and volume

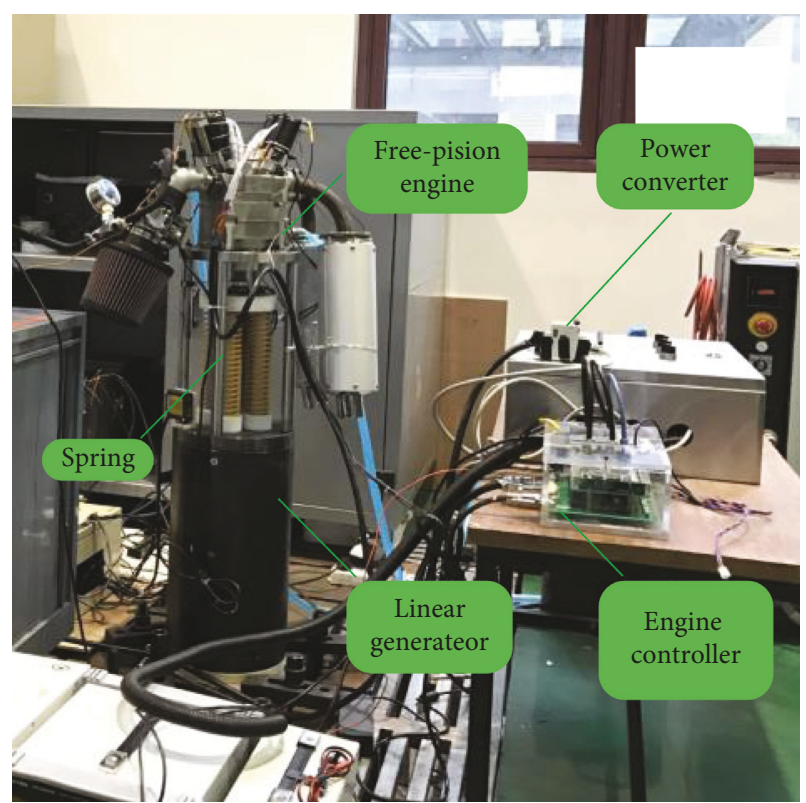

Figure 6: Experiment system of FEPG.

are in accordance with the ideal gas state equation. (3) The mass of the gas in the cylinder is constant and the flow leakage in the gas exchange process is ignored. So the one-dimensional dynamics model in a pipe is described by three equations.

The energy equation:

$$
\begin{aligned}
\frac{\partial E}{\partial t}= & \frac{-\partial[u \cdot(P+E)]}{\partial x}-u \cdot(P+E) \\
& \cdot \frac{1}{A} \cdot \frac{\partial A}{\partial x}+\frac{q_{w}}{V} \\
V= & A \cdot d x \\
E= & \rho \cdot \bar{C}_{v} \cdot T+\frac{1}{2} \cdot \rho \cdot u^{2} .
\end{aligned}
$$

The equation for the conservation of momentum:

$$
\frac{\partial(\rho \cdot u)}{\partial t}=\frac{-\partial\left(\rho \cdot u^{2}+P\right)}{\partial x}-\rho \cdot u^{2} \cdot \frac{1}{A} \cdot \frac{\partial A}{\partial x} \cdot \frac{F_{R}}{V} .
$$

The continuity equation of the working medium:

$$
\frac{\partial \rho}{\partial t}=\frac{-\partial(\rho \cdot u)}{\partial x}-\rho \cdot u \cdot \frac{1}{A} \cdot \frac{d A}{d x},
$$

where $E$ represents the energy content of the ideal gas, $u$ represents the flow velocity, $P$ represents the static pressure, $A$ represents the cross-sectional area of the pipe, $q_{w}$ represents the heat flow of the wall, $V$ represents the unit volume, $\rho$ represents the density of the working medium, $\bar{C}_{v}$ represents the specific heat in a content volume, and $F_{R}$ represents the friction force between the fluid and the pipe wall.

3.2. Gas Pressure in the Cylinder. According to the assumptions above, we also assumed that the in-cylinder gas pressure is equal to intake pressure $P_{\mathrm{C} 0}$ and so is the exhaust stroke. When the volume of the combustion chamber is zero, 
the piston position is set as the origin of displacement. Using the first law of thermodynamics and the ideal gas-state equation, the in-cylinder gas pressure can be written as the following equation:

$$
\frac{\partial P}{\partial t}=\frac{1}{V}\left[(\gamma-1) \cdot \frac{d Q}{d t}-\gamma \cdot p \cdot \frac{d V}{d t}\right],
$$

where $P$ represents the in-cylinder gas pressure, $V$ represents the volume of cylinder, $\gamma$ represents the specific heat ratio of the working medium, and $Q$ represents the heat release rate of fuel.

3.3. Combustion in the Cylinder. The exothermic characteristic of free-piston engine is determined by the flame propagation velocity and the shape of the combustion chamber. In this paper, the simulation model adopts a zero-dimensional single-zone combustion model, which defined the whole combustion chamber as a closed space and ignored the flow leakage. A Weber function can be used to represent the actual combustion process and express the heat release. The heat released by the combustion process is as follows:

$$
\begin{aligned}
\frac{d Q}{d t}= & 6.908 \cdot H_{\mathrm{u}} \cdot G_{\mathrm{u}} \cdot \eta \cdot \frac{n+1}{T} \cdot\left(\frac{t-t_{\mathrm{c}}}{T}\right)^{n} \\
& \cdot e^{\left[-6.908\left(\left(t-t_{\mathrm{c}}\right) / T\right)\right]^{n+1}},
\end{aligned}
$$

where $Q$ represents the heat release rate of the fuel, $H_{\mathrm{u}}$ represents the lower calorific value of the fuel, $G_{\mathrm{u}}$ represents the injected fuel mass per cycle, $\eta$ represents the combustion efficiency, $n$ represents the combustion quality index, $T$ represents the combustion duration, and $t$ represents the time variable, $t_{c}$ represents the start time of combustion.

3.4. Heat Transfer from the Cylinder. During calculating the heat transfer loss, the heat transfer irreversibility of reciprocating heat cycle is nonnegligible. It is assumed that the losses happened only in the combustion and expansion strokes, and the heat transfer from the combustion chamber to outside is negligible. From the in-cylinder gases to cylinder walls, the calculation equation of the heat transfer is

$$
\frac{d Q}{d t}=\alpha_{\mathrm{w}} \cdot \pi \cdot D \cdot(0.5 D+x) \cdot\left(T_{\mathrm{w}}-T\right),
$$

where $Q$ represents the heat release rate of the fuel, $\alpha_{w}$ represents the heat transfer coefficient, $D$ represents cylinder diameter, $x$ represents the piston position, $T_{\mathrm{w}}$ represents the temperature of the cylinder walls, and $T$ represents the incylinder gas temperature.

Hereinto, the calculation equation adopts the heat transfer function of Woschni 1978. The function is suitable for the high-pressure cycle, and the heat transfer coefficient is

$$
\begin{aligned}
& \alpha_{\mathrm{w}}=820 D^{-0.2} P_{\mathrm{c}}^{0.8} T_{\mathrm{c}}^{-0.53} \times\left(C_{1} C_{\mathrm{m}}\right)^{0.8}, \\
& C_{1}=2.28+0.308 \times \frac{C_{\mathrm{u}}}{C_{\mathrm{m}}},
\end{aligned}
$$

where $D$ represents the cylinder diameter, $P_{c}$ represents the in-cylinder gas pressure, $T_{c}$ represents the in-cylinder gas temperature, $C_{\mathrm{u}}$ represents the circular speed, and $C_{\mathrm{m}}$ represents the mean velocity of piston.

3.5. Simulation Model. During the process of establishing the FPEG model, the one-dimensional simulation model is mainly divided into two parts. The first part included the dimensional parameters of the engine, such as cylinder diameter, the length of intake pipe, and exhaust pipe. The other part contained the thermodynamic model, combustion model, and the heat transfer model.

FPEG modeling steps areas follows [20]: (1) study the main measurement parameters of the engine and collect the data and information of the structure. (2) Divide the actual freepiston engine into several easy-to-operate subsystems, and use of AVL BOOST submodules to establish the corresponding physical submodels. (3) According to the theoretically knowledge of dynamics, heat transfer, thermodynamics, combustion, a simple physical model has been built, which contains the collected data and the input information for the engine submodule. (4) Use the established model to accomplish the elementary simulation and find the physical parameters of simulation model in order to modify the error.

Based on the theoretical analysis and mathematic model above, the one-dimensional simulation model of the FPEG is established in AVL BOOST software to simulate the fourstroke thermodynamic cycle and two-stroke thermodynamic cycle. As we all know, a complete simulation model of the FPEG system should include intake system, combustion system, and exhaust system. According to the structure parameters above and experiment system in the previous section, the one-dimensional flow simulation model is established as shown in Figure 7.

3.6. Simulation Parameters. Before running the simulation model, the key step is to select the control parameters. The initial value of boundary conditions includes pressure, temperature, and air-fuel ratio. Thereinto, the cylinder parameters contains bore, stroke, connecting rod length, and compression ratio. The control parameters of heat transfer and valve specification also need to be determined. Table 2 lists the specific parameters of each component.

\section{Model Validation}

The free-piston trajectory of the FPEG during a four-stroke working cycle is simulated. As shown in Figure 8, the working period of the four-stroke free-piston engine is about $100 \mathrm{~ms}$. It is clear that the piston displacement is asymmetric, the intake and compression strokes are shorter than expansion and exhaust strokes. The expansion ratio is greater than the compression ratio, and the longer expansion and exhaust are beneficial to achieve full expansion and reduce residual gas. Therefore, the characteristics of the FPEG are different from the conventional engine, and it has 


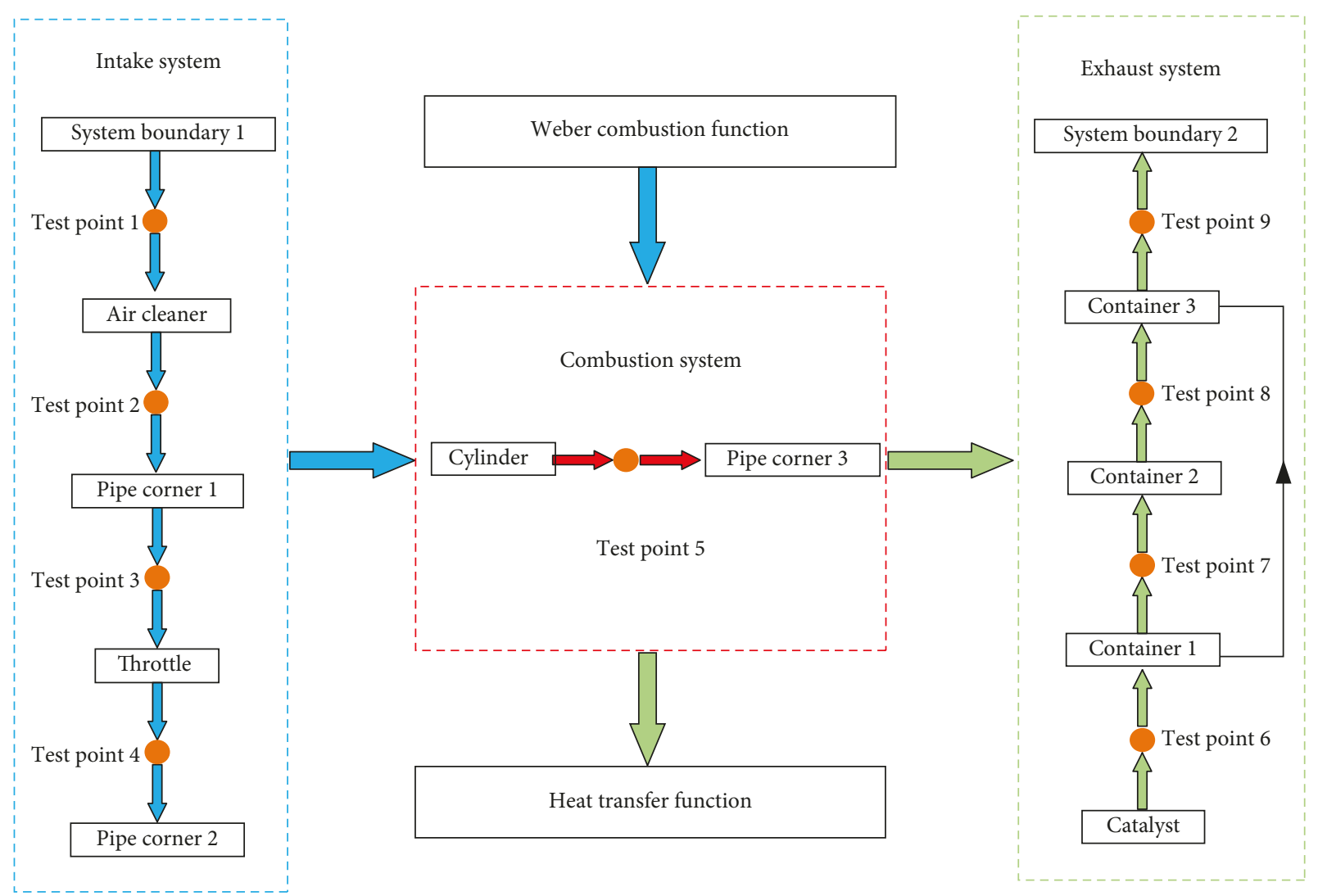

FIgURE 7: The one-dimensional simulation model of the FPEG system.

TABLE 2: The specific parameters of the simulation model.

\begin{tabular}{lcc}
\hline Components & Parameters & Value \\
\hline Air cleaner & Total volume & $3.1 \mathrm{~L}$ \\
Throttle & Throttle angle & $18.5^{\circ}$ \\
& Bore & $102 \mathrm{~mm}$ \\
Cylinder & Stroke & $126 \mathrm{~mm}$ \\
& Connecting rod length & $150 \mathrm{~mm}$ \\
& Compression ratio & 9.3 \\
Intake valve & Valve opening & $48.5 \mathrm{~ms}$ \\
& Valve closing & $50.2 \mathrm{~ms}$ \\
Exhaust valve & Valve opening & $19.1 \mathrm{~ms}$ \\
Catalyst & Valve closed & $23.4 \mathrm{~ms}$ \\
System boundary 1 & Monolith volume & $0.3 \mathrm{~L}$ \\
& Pressure & $1.1 \mathrm{bar}$ \\
System boundary 2 & Gas temperature & $24.85^{\circ} \mathrm{C}$ \\
& Pressure & $1.0 \mathrm{bar}$ \\
& Gas temperature & $126.85^{\circ} \mathrm{C}$ \\
\hline
\end{tabular}

the great benefit in terms of fuel efficiency and emissions formation.

A four-stroke experiment is completed on the FPEG system to validate the simulation model. As seen from Figure 9, it compares the in-cylinder pressure of test data with simulation results during a four-stroke working cycle, which is obtained by an in-cylinder pressure sensor. Compared with the experimental results, the in-cylinder pressure curves of the test and simulation are coincident; the maximum deviation of in-cylinder pressure variation is $5.2 \%$, and the average deviation is $1.5 \%$. Table 3 lists the comparison results of FPEG

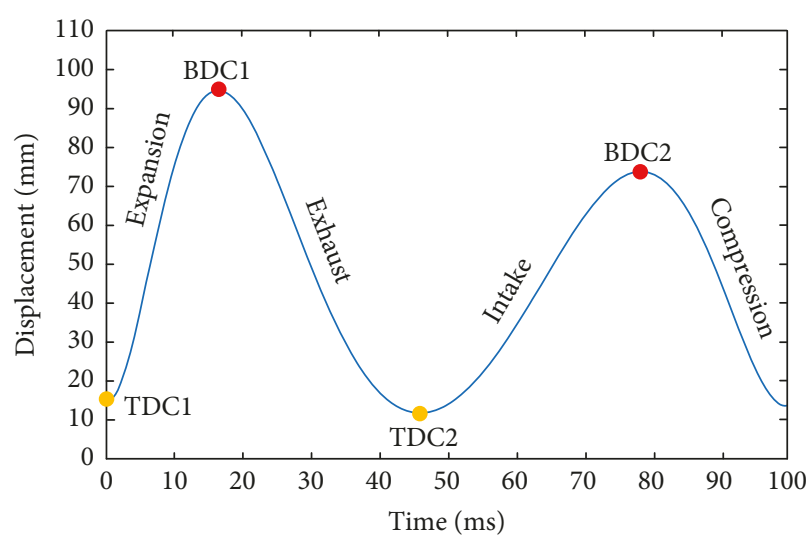

FIgURE 8: The four-stroke motion trajectory of FPEG.

performance. Thus, the simulated results meet the requirements of the accuracy, and we believe that the simulation model is an accurate model of the FPEG. Moreover, in the FPEG system, the start time of the combustion is $-3.1 \mathrm{~ms}$ and the combustion duration is $6.4 \mathrm{~ms}$, which are determined by the four-stroke experimental results.

\section{Two-Stroke Thermodynamic Cycle Optimization}

The simulated motion curve of free-piston during a twostroke working cycle is shown in Figure 10. As can be seen, 


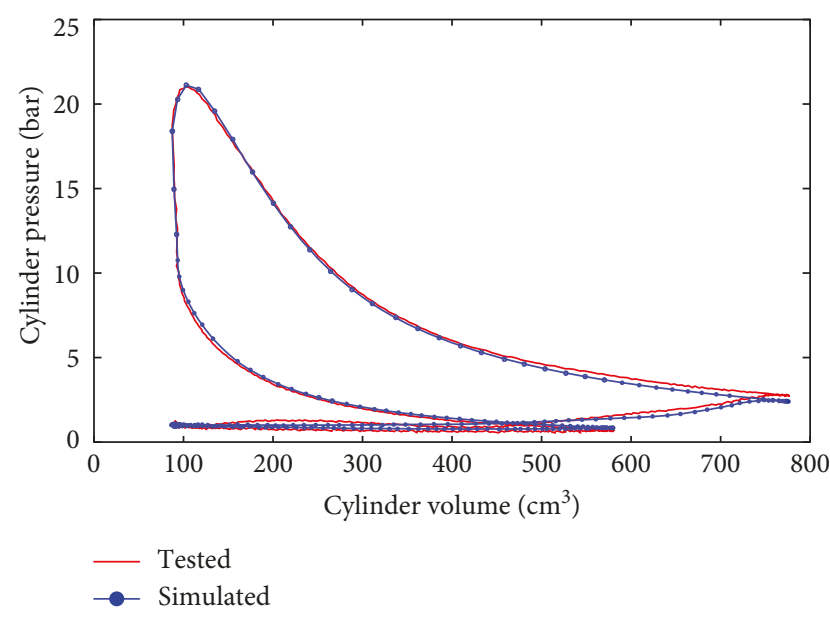

FIGURE 9: Tested and simulated FPEG in-cylinder pressure.

TABLE 3: Comparison of test and simulation results.

\begin{tabular}{lccc}
\hline Name & Unit & Test & Simulation \\
\hline Effective power & $\mathrm{kW}$ & 4.75 & 4.82 \\
Peak pressure & $\mathrm{bar}$ & 21.02 & 21.40 \\
Residual gas content & - & 0.0809 & 0.0769 \\
Start of combustion & $\mathrm{ms}$ & -3.1 & -3.1 \\
Combustion duration & $\mathrm{ms}$ & 6.4 & 6.4 \\
\hline
\end{tabular}

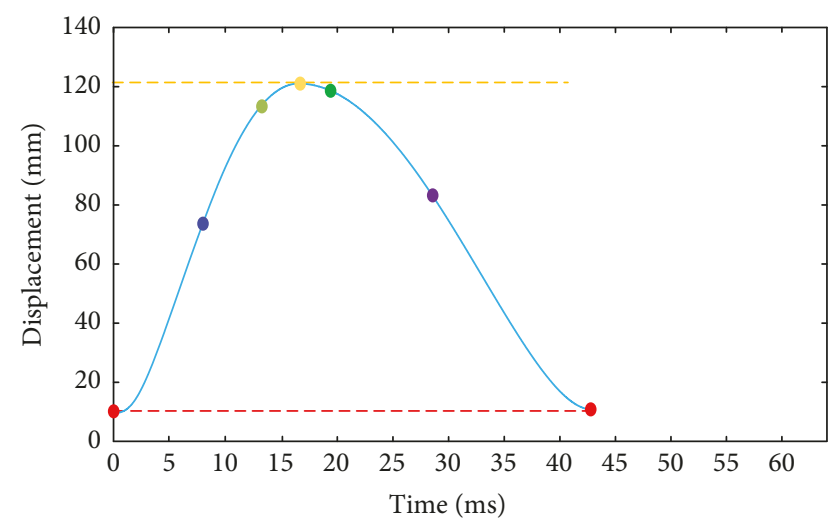

timing, intake valve timing, and exhaust valve timing. Then, the influence of thermodynamic cycle is analyzed, and the thermodynamic performance of FPEG is optimized.

5.1. Influence of Intake Pressurization. Research shows that the improvement of intake air pressure can ensure a good combustion state. The FPEG model is simulated under different intake air pressure, and the changing curves are as follows. Indicated power, residual gas coefficient, indicated specific fuel consumption (ISFC), and intake flow are the main evaluation indexes, and they can be found in simulation results. According to the pressurize range of an actual turbocharger, the range of the intake pressurization is 1.0 bar 1.4 bar.

Figure 11 depicts the indicated power and intake flow gradually increased, the residual gas coefficient gradually decreased in the range of intake pressurization, and the four evaluation indexes change more obviously in the range of 1.0 bar 1.1 bar. The results show that two-stroke free-piston engine cannot obtain enough intake air flow to complete the work cycle in a normal intake air pressure. This is because more fuel mixture can be absorbed to the cylinder as higher intake air pressure is provided. Furthermore, the higher intake air pressure provided a large compression pressure. Therefore, the increasing of intake air pressure results in improving the indicated power and fuel economy.

5.2. Influence of Injection Timing. In the combustion system of the one-dimensional simulation model, the parameters of injection timing can be changed to simulate its effect on FPEG performance. As can be seen, Figure 12 depicts the influence of different injection time. The mean effective pressure (MEP) is the effective power generated by the working volume per unit cylinder, and it is an important index for evaluating the power performance.

The range of injection time is divided into three parts, $0 \mathrm{~ms} \sim 7.2 \mathrm{~ms}, 7.2 \mathrm{~ms} \sim 14.4 \mathrm{~ms}$, and $14.4 \mathrm{~ms} \sim 21.6 \mathrm{~ms}$. Firstly, the indicated power and MEP are maintained at a low-level of fluctuation, and the residual gas coefficient remains unchanged at a higher-level. Because the process of fuel injection has completed before the intake valve opening, most of the fuel mixture failed to enter the combustion chamber. Secondly, the injection time and intake process are consistent, and the thermodynamic performance of FPEG has enhanced significantly to improve indicated power and ventilation efficiency. Finally, compared with the first part, all the performance values are similar in the range of $14.4 \mathrm{~ms}$ to $21.6 \mathrm{~ms}$. This is because the injection time leaves behind intake process and part of the fuel mixture cannot be used in the combustion process. As can be seen, the engine performance achieved optimal results at the moment of $14.4 \mathrm{~ms}$.

5.3. Influence of Ignition Timing. The effect of advanced ignition is to start burning ahead before the piston moves to TDC. When the piston moves to TDC and enters the expansion stroke, the mixture-working medium completely burned and released more energy. Therefore, the range of 


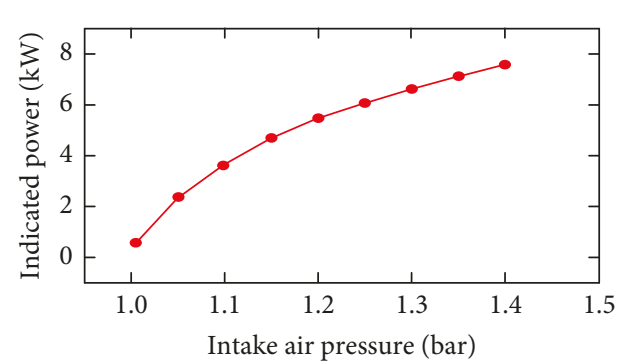

(a)

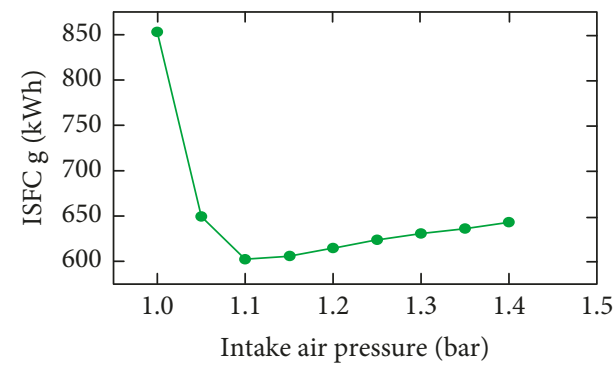

(c)

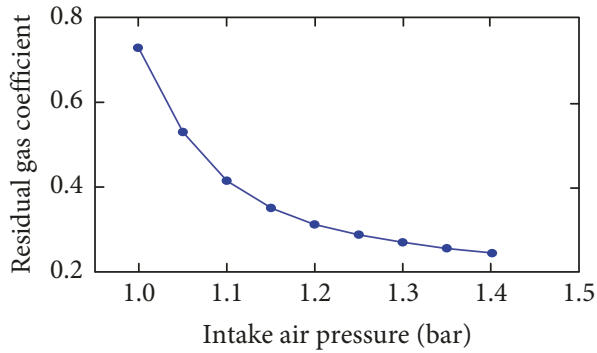

(b)

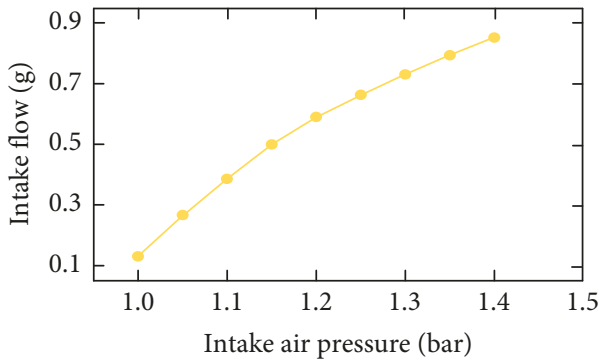

(d)

FIGURE 11: The influence of intake pressurization.

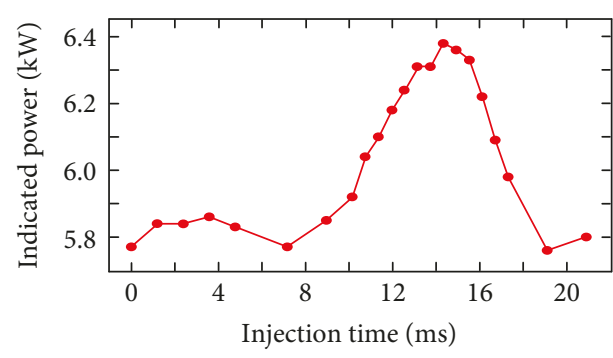

(a)

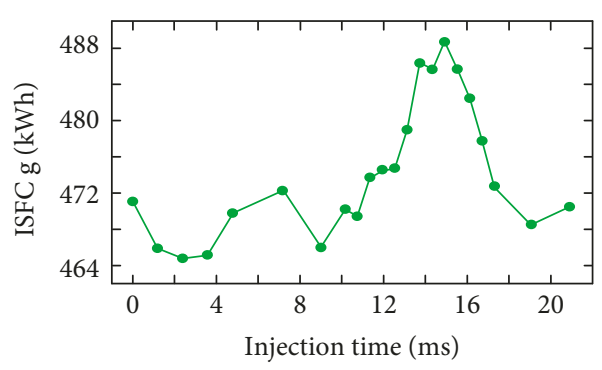

(c)

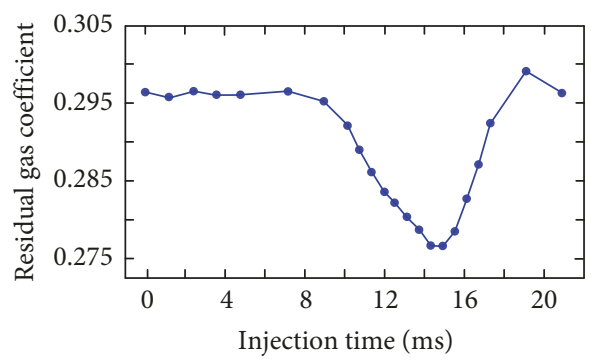

(b)

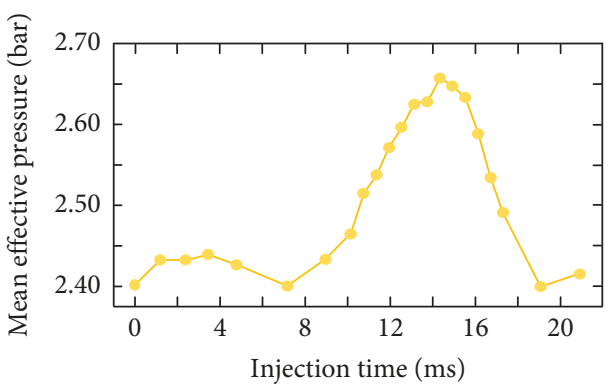

(d)

FIgURE 12: The influence of injection timing.

ignition time is $-5.4 \mathrm{~ms}$ to $0 \mathrm{~ms}$, and the simulation results are shown in Figure 13.

In the range of $-3 \mathrm{~ms}$ to $-5.4 \mathrm{~ms}$, the indicated power and MEP gradually decreased, and the ISCF gradually increased. This is because the mixture ignited prematurely and the burning gas expanded. Part of energy prevents the piston from moving upward to TDC. Then, the indicated power and MEP gradually decreased with different ignition time, and the ISFC gradually increased in the range of $-3 \mathrm{~ms}$ to $0 \mathrm{~ms}$. Due to the delay of ignition time, the piston moves downward before the mixture starts to burn. It leads to a larger cylinder volume and the decreasing combustion pressure, and the thermodynamic performance of FPEG is in the state of high fuel consumption and low output power. Besides, the engine performance achieved optimal results at $-3 \mathrm{~ms}$.

5.4. Influence of Intake Valve Timing. Under the condition of keeping valve lift and valve opening duration unchanged, the FPEG model is simulated under different intake opening 


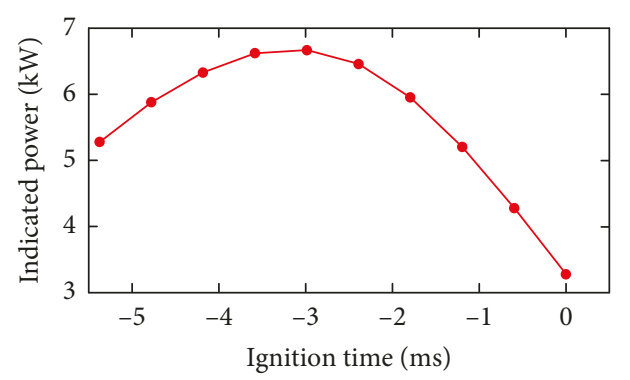

(a)

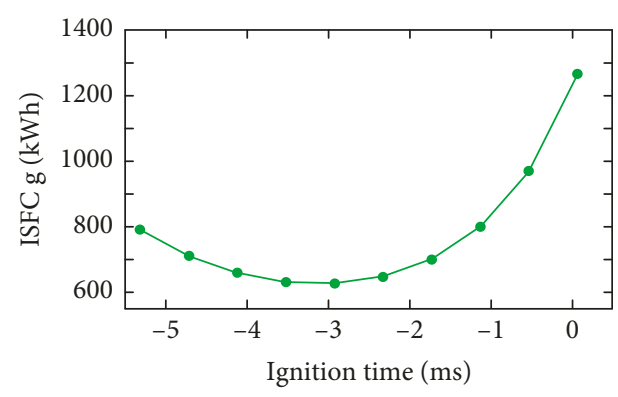

(c)

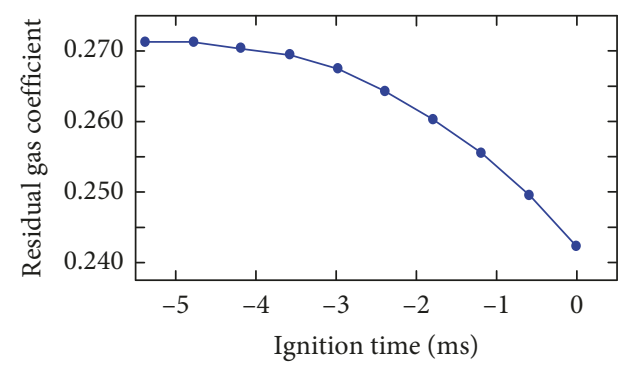

(b)

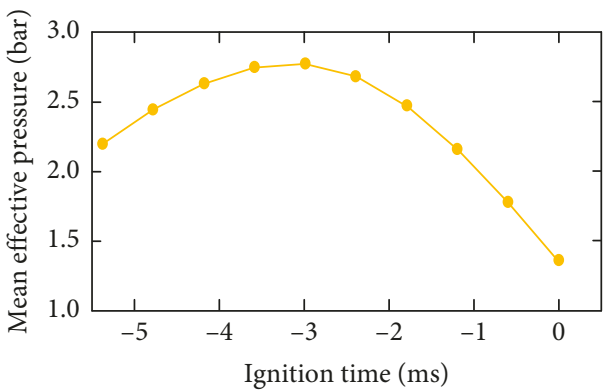

(d)

FIGURE 13: The influence of ignition timing.

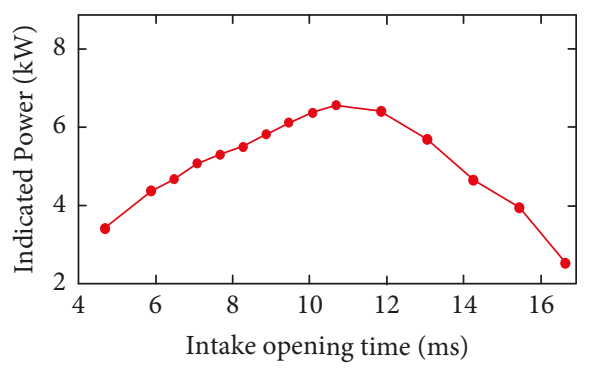

(a)

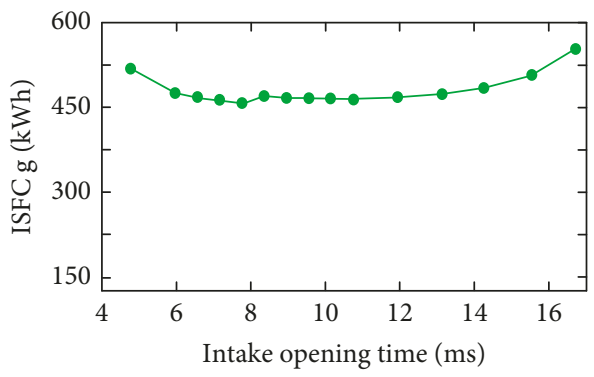

(c)

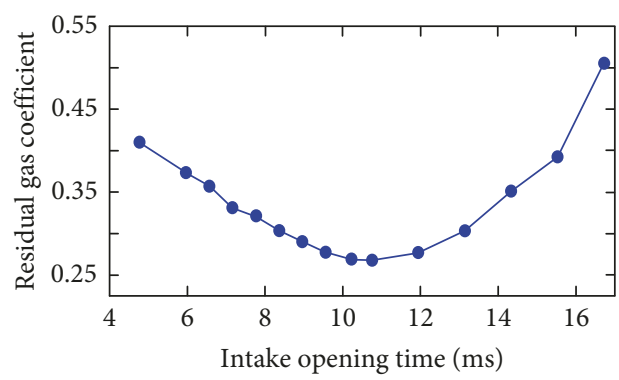

(b)

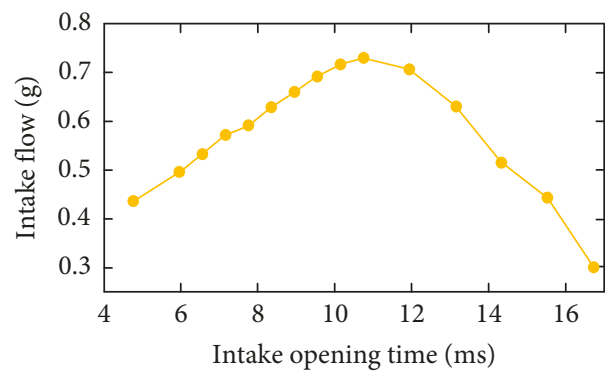

(d)

Figure 14: The influence of intake opening timing.

time. As shown in Figure 14, with the intake opening time from $4.8 \mathrm{~ms}$ to $16.8 \mathrm{~ms}$, the indicated power and intake flow show an overall trend of rising first then falling and get the maximum value at $10.8 \mathrm{~ms}$. Meanwhile, the characteristics of the residual gas coefficient and ISFC are contrary to the change law of intake flow.

When the intake opening time is in the range of $4.8 \mathrm{~ms}$ to $10.8 \mathrm{~ms}$, the intake and exhaust valves open simultaneously.
It creates a scavenging flow in the cylinder, which makes the process of gas exchange more complete and reduces the residual gas. After that, the time of intake valve opening is late and part of the fuel mixture failed to enter the cylinder, thus the combustion process is insufficient and the thermodynamic performance decreased significantly. Due to the fixed time of valve duration, the optimal period of intake valve opening is from $10.8 \mathrm{~ms}$ to $24.5 \mathrm{~ms}$. 


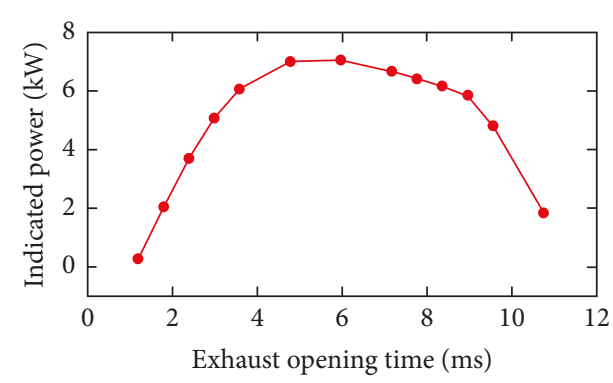

(a)

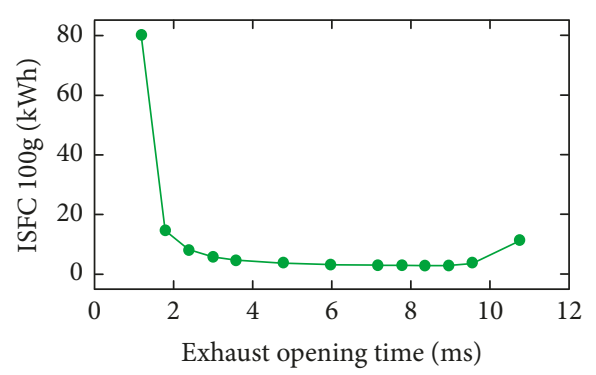

(c)

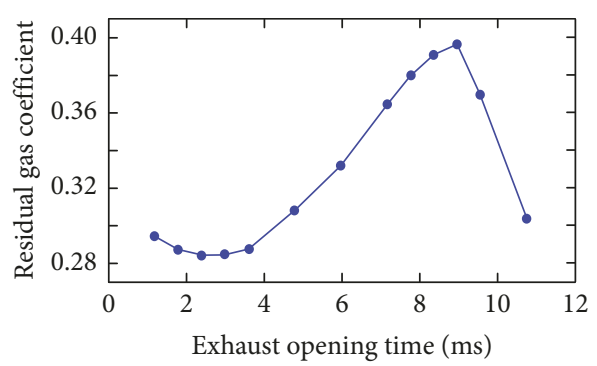

(b)

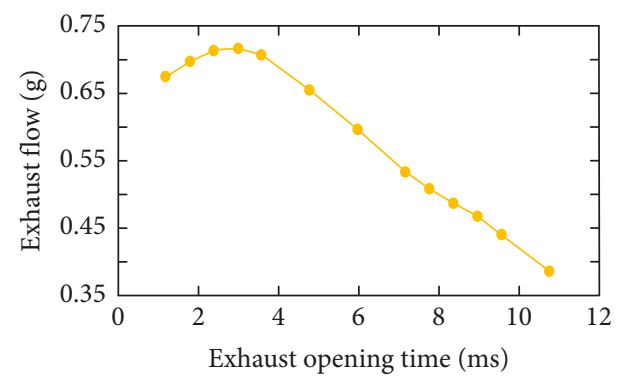

(d)

Figure 15: The influence of exhaust opening timing.

5.5. Influence of Exhaust Valve Timing. As described in Figure 15, with the change of exhaust opening time from $1.2 \mathrm{~ms}$ to $10.8 \mathrm{~ms}$, the indicated power and exhaust flow show an overall trend of rising first then falling. The exhaust flow increased in the range of $1.2 \mathrm{~ms}$ to $3.6 \mathrm{~ms}$ and then gradually decreased, and it gets the maximum value at $3.6 \mathrm{~ms}$. The indicated power, residual gas coefficient, and ISCF improved with the increase of exhaust flow.

The results show that the premature opening of exhaust valve leads to an insufficient combustion process and the power performance and fuel economy of FPEG will be reduced. With the delay of exhaust valve opening, the residual gas in the cylinder cannot be expelled completely, and it will affect the next cycle of combustion. Therefore, the proper opening time of the exhaust valve has great improvement on the FPEG performance, and the optimal period of the exhaust valve opening is from $3.6 \mathrm{~ms}$ to $23.1 \mathrm{~ms}$.

5.6. Optimized Performance of the FPEG. According to the above simulation results, we have made adjustments for the control parameters of the FPEG model. The adjusted parameters include ignition time, injection time, and valve opening time. The adjusted model has been simulated at the working frequency of $25 \mathrm{~Hz}$, namely, 25 reciprocating cycles per second. The optimized results show that the indicated thermal efficiency is about $27.6 \%$, the indicated power is $6.7 \mathrm{~kW}$, and the ISFC is $481.6 \mathrm{~g} / \mathrm{kWh}$. The specific results of the FPEG thermodynamic performance for two-stroke thermodynamic cycle are shown in Table 4.

\section{Conclusions}

The work presented the two-stroke thermodynamic performance optimization of a single-cylinder FPEG. The
TABle 4: Optimized thermodynamic performance of FPEG.

\begin{tabular}{lcc}
\hline Items & Unit & Value \\
\hline Indicated power & $\mathrm{kW}$ & 6.7 \\
Indicated thermal efficiency & $\%$ & 27.6 \\
Indicated specific fuel consumption (ISCF) & $\mathrm{g} / \mathrm{kWh}$ & 481.6 \\
Residual gas content & - & 0.285 \\
Mean effective pressure & $\mathrm{bar}$ & 2.6 \\
Cycle intake mass & $\mathrm{g}$ & 0.671 \\
\hline
\end{tabular}

comprehensive one-dimensional flow simulation model of the FPEG is established, and the accuracy of the model is validated by the experimental results tested from the FPEG prototype. The four-stroke experimental results manifested the effective power of $4.75 \mathrm{~kW}$, and the peak pressure of 21.02 bar has been obtained. On this basis, the two-stroke thermodynamic cycle has been simulated and optimized. The simulation results show that the indicated thermal efficiency of FPEG is about $27.6 \%$, and the indicated power of $6.7 \mathrm{~kW}$ can be achieved at the working frequency of $25 \mathrm{~Hz}$. From these results, we conclude that the thermodynamic performance of high efficiency and energy saving for the FPEG system can be significantly promoted by optimizing the two-stroke thermodynamic cycle.

In the future, an experimental test will be implemented to validate the simulation results of two-stroke thermodynamic cycle optimization in this paper. Furthermore, the two-stroke free-piston engine generator will be investigated by the multiobjective intelligent optimization to obtain higher output power and effective efficiency.

\section{Data Availability}

The data used to support the findings of this study are available from the corresponding author upon request. 


\section{Conflicts of Interest}

The authors declare that there are no conflicts of interest regarding the publication of this paper.

\section{Acknowledgments}

The authors would like to appreciate the National Natural Science Foundation of China (Grant no. 51875290).

\section{References}

[1] F. Kara and B. Öztürk, "Comparison and optimization of PVD and CVD method on surface roughness and flank wear in hard-machining of DIN 1.2738 mold steel," Sensor Review, vol. 39, no. 1, pp. 24-33, 2019.

[2] B. Öztürk, L. Uğur, and A. Yildiz, "Investigation of effect on energy consumption of surface roughness in $\mathrm{X}$-axis and spindle servo motors in slot milling operation," Measurement, vol. 139, pp. 92-102, 2019.

[3] N. B. Hung and O. Lim, "A review of free-piston linear engines,” Applied Energy, vol. 178, pp. 78-97, 2016.

[4] E. Nas and B. Öztürk, "Optimization of surface roughness via the Taguchi method and investigation of energy consumption when milling spheroidal graphite cast iron materials," $\mathrm{Ma}$ terials Testing, vol. 60, no. 5, pp. 1136-1143, 2009.

[5] P. Sun, C. Zhang, J. Chen et al., "Decoupling design and verification of a free-piston linear generator," Energies, vol. 9, no. 12 , p. $1067,2016$.

[6] N. N. Clark, T. L. McDaniel, and R. J. Atkinson, "Modeling and development of a linear engine," in Proceedings of the ASME Spring Conference: Internal Combustion Engine Division, pp. 49-57, Clymer, NY, USA, December 1998.

[7] P. Famouri, W. R. Cawthorne, N. Clark, S. Nandkumar, and C. Atkinson, "Design and testing of a novel linear alternator and engine system for remote electrical power generation," in Proceedings of the IEEE Power Engineering Society Winter Meeting, pp. 108-112, New York City, NY, USA, January 1999.

[8] Y. Ö. Özgören, S. Çetinkaya, S. Sarıdemir, A. Çiçek, and F. Kara, "Artificial neural network based modelling of performance of a beta-type stirling engine," Proceedings of the Institution of Mechanical Engineers, Part E: Journal of Process Mechanical Engineering, vol. 227, no. 3, pp. 166-177, 2016.

[9] Y. Ö. Özgören, S. Çetinkaya, S. Sarıdemir, A. Çiçek, and F. Kara, "Predictive modeling of performance of a helium charged stirling engine using an artificial neural network," Energy Conversion and Management, vol. 67, pp. 357-368, 2013.

[10] K. Moriya, S. Goto, T. Akita, H. Kosaka, Y. Hotta, and K. Nakakita, "Development of free piston engine linear generator system Part 3-novel control method of linear generator for to improve efficiency and stability," in Proceedings of the SAE Technical Paper, pp. 1-685, Detroit, MI, USA, April 2016.

[11] Z. Xu and S. Chang, "Prototype testing and analysis of a novel internal combustion linear generator integrated power system," Applied Energy, vol. 87, no. 4, pp. 1342-1348, 2010.

[12] Z. Xu and S. Chang, "Hierarchical hybrid control of a fourstroke free-piston engine for electrical power generation," in Proceedings of the International Conference on Mechatronics and Automation, pp. 4045-4049, Changchun, China, August 2009.

[13] S. Goto, K. Moriya, and H. Kosaka, "Development of free piston engine linear generator system," in Proceedings of the
SAE Technical Paper, vol. 1, p. 1193, Detroit, MI, USA, April 2014.

[14] Z. Zhang, X. Chen, and Z. Xu, "A control strategy of freepiston linear generator for reducing the fuel consumption per unit power," Energies, vol. 11, no. 1, p. 135, 2018.

[15] Z. Xu and S. Chang, "Improved moving coil electric machine for internal combustion linear generator," IEEE Transactions on Energy Conversion, vol. 25, no. 2, pp. 281-286, 2010.

[16] Z. Zhao and D. Wu, "Experimental investigation of the cycleto-cycle variations in combustion process of a hydraulic freepiston engine," Energy, vol. 78, pp. 257-265, 2014.

[17] J. Lin, Z. Xu, S. Chang, and N. Yin, "Thermodynamic simulation and prototype testing of a four-stroke free-piston engine," Engineering for Gas Turbines and Power, vol. 136, no. 5, article 051505, 2014.

[18] X. Pei, A. Smith, D. Shuttle worth, and M. Barnes, "Hybrid system modeling and full cycle operation analysis of a twostroke free-piston linear generator," Energies, vol. 10, no. 2, p. 213, 2017.

[19] H. Feng, Y. Guo, Y. Song, C. Guo, and Z. Zuo, "Study of the injection control strategies of a compression ignition free piston engine linear generator in a one-stroke starting process," Energies, vol. 9, no. 6, p. 453, 2016.

[20] B. Qin, "Simulation analysis of piston engine based on BOOST," in Proceedings of International Conference on Mechanical and Electrical Technology, Singapore, July 2010. 


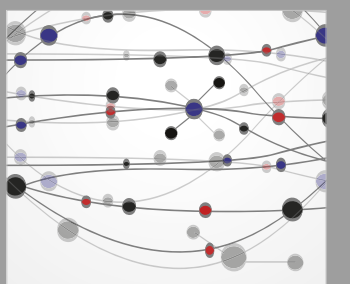

The Scientific World Journal
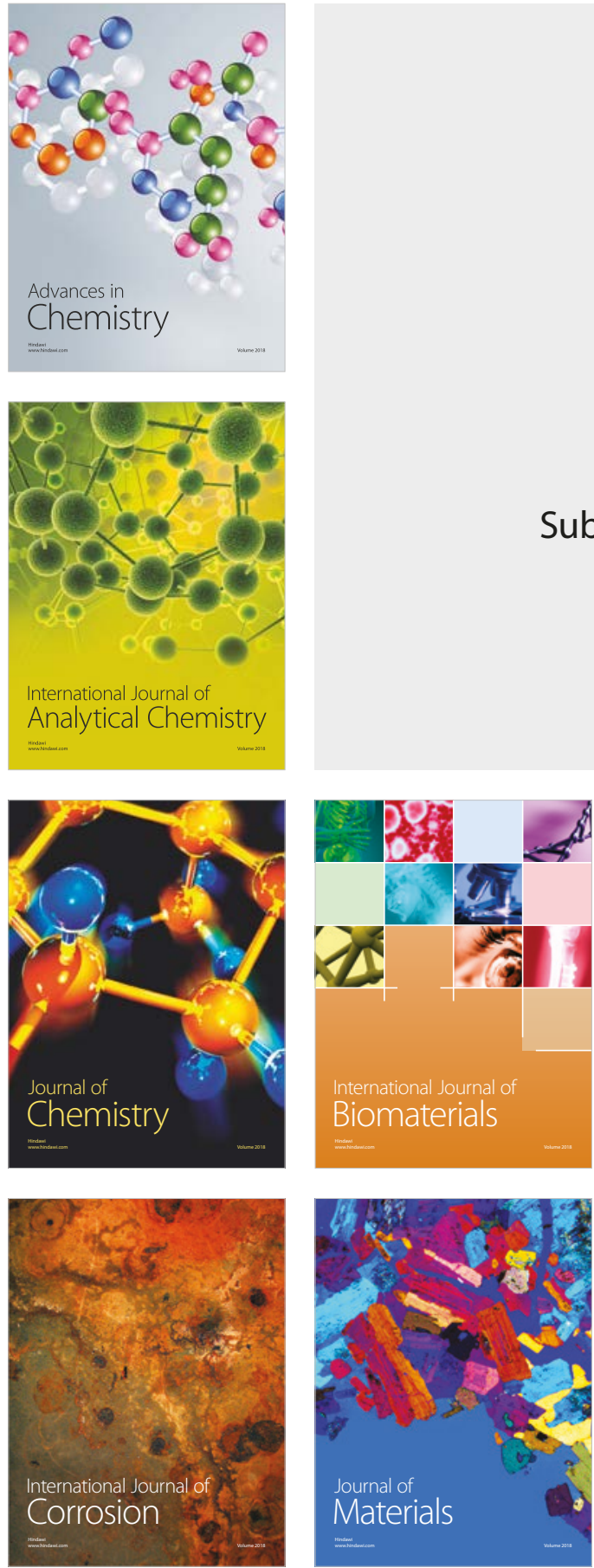

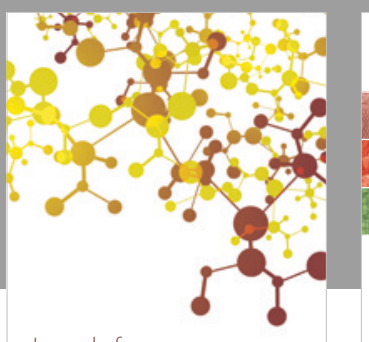

Journal of

Applied Chemistry
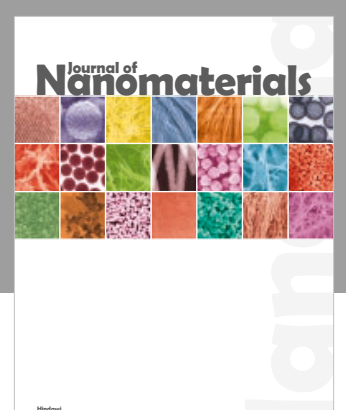

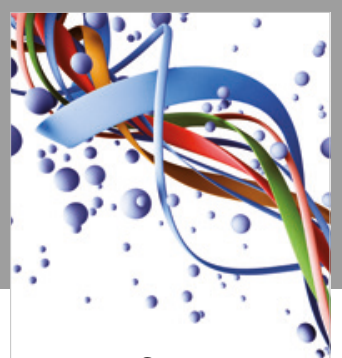

Scientifica

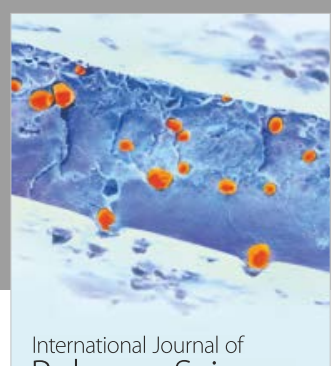

Polymer Science

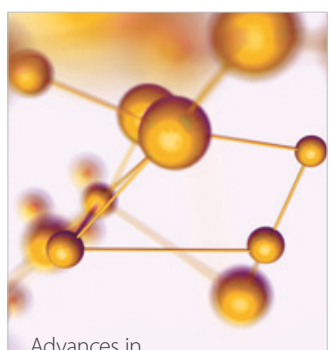

Physical Chemistry
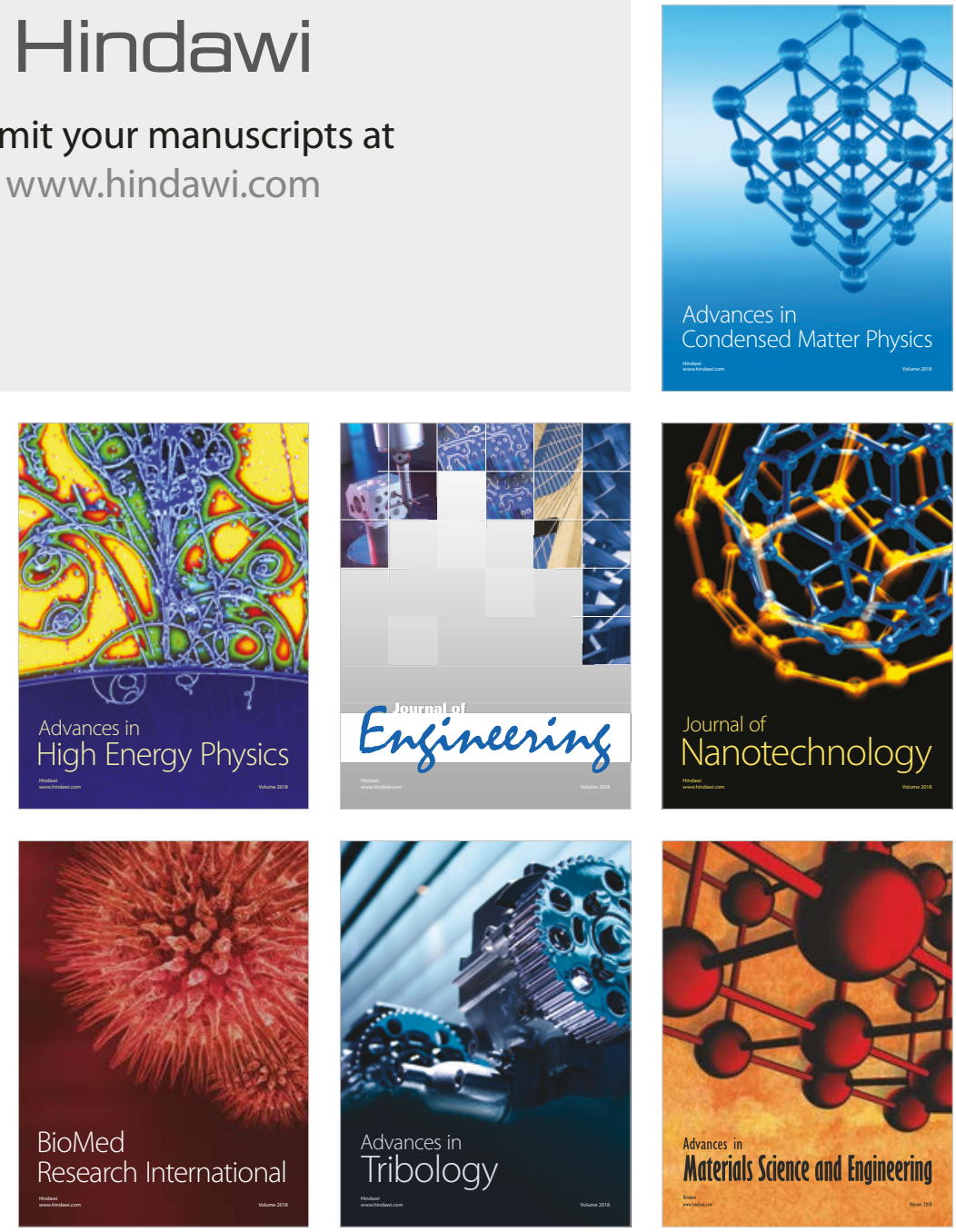